\title{
Geostatistische Auswertungsmöglichkeiten für Waldschadeninventuren: Methodische Überlegungen zur Beschreibung räumlicher Verteilungen
}

\author{
Von M. KöhL und G. Gertiner
}

\section{Einleitung}

Die schweizerische Waldschadeninventur (WSI) wird seit 1985 jährlich zur Beschreibung des Gesundheitszustandes des Waides der gesamten Schweiz durchgeführt. Auf den Schnittpunkten eines $4 \times 4 \mathrm{~km}$ Netzes werden auf zwei konzentrischen, permanenten Probekreisen die Nadel-/Blatverluste (NBV) der Finzelbäume in $5 \%$-Stufen erfaßt. Die räumliche Verteilung der Nadel-/Blattverluste wurde bisher nicht dargestellt. Die Angaben in den WSI-Berichten beschränken sich auf Durchschnittswerte und Anteile für die einzelnen Befundeinheiten. Hier wird versucht, mit Hilfe geostatistischer Methoden ausgehend ron den durchschnittichen Nadel-/Blattverlusten der einzelnen Probeflächen die räumliche Verteilung der Nadel-/Blattverluste darzustellen. Ergebnisse werden für die Jahre 1986 und 1990 präsentiert.

Die Anwendung geostatistischer Methoden ist notwendig, da die beobachteten Nadel-I Blattverluste der einzelnen Probeflächen nicht repräsentativ für die sie umgebende, $16 \mathrm{~km}^{2}$ große Fläche sind. Eine einfache Karte der beobachteten Nadel-/Blattverluste fuhrt somic zwangsläufig zu einer verfälschten Darstellung der räumlichen Verteilungs. Die Interpretation einer solchen Rohdatenkarte ist kaum möglich, da nicht unterschieden werden kann, ob die Lokalisation eines Nadel-/Blattverlustes einen regionalen Trend oder eine zufällige Beobachtung innerhalb der kleinräumlichen Variation darstellt.

Geostatistische Methoden haben ihren Ursprung im Bereich der Mineralogie und der Lagerstättenerkundung, wo sie in den $60 \mathrm{er}$ Jahren eingetührt wurden und sich mittlerweile zu einem bewährten Instrument bei der Erhebung von Ressourcen entwickelt haben.

Im folgenden wird eine kurze Einführung in die geostatistischen Methoden und die Probleme bei der Anwendung auf die Nadel-/Blattverlust-Schätzung der WSI gegeben. Das Potential, das geostatistische Methoden zur Ursachenanalyse bieten, sowie Ansätze tür ein integrales Unwelmonitoring werden aufgezeigr.'

\section{Methode}

Dic Entwicklung der geostatistischen Methoden, auch räumliche Statistili genannt, läßt sich im wesentlichen auf zwei Personen zurückführen, die beide im Bereich des Bergbaus beheimatet sind. G. Matheron (1965) hat in Frankreich die theoretische Formulierung der räumlichen Statistik initjert, die allgemein als die Theorie der Regionalisierten Variablen bezeichnet wird. In Südafrika hat D. G. KRIGE $(1951$, 1966) geostatistische Methoden empirisch entwickelt und bei der Erhebung von Goldlagerstätten angewendet. Mitrlerweile existieren einige umfassende Darstellungen der Geostatistik (z. B. CLARK [1979]; David

\footnotetext{
${ }^{1}$ Wir danken Frau Dipl.-Forstwirtin A. Jost, Abccilung Forstliche Biometrie der Liniversitäe Freiburg i. Br., für die vielen hilfreichen Hinweise sorwie die kritisuhe Durchsicht des Mhnuskripts.
} 
[1977]; Journel u. Hutjbregts [1978]; Akin u. Siemes [1988]; Webster u. Olver [1990]).

Bei klassischen Stichprobeninventuren wird unterstellt, daß einzelne Stichprobenelemente - oder kurz Proben -- aus der Grundgesamtheit zufällig entnommen werden. Die räumliche Position der Proben wird bei der Herleitung von Gesamtaussagen für die Befundeinheiten vernachlässigt. Intuitiv ist allerdings $\mathrm{klar}$, daß räumlich benachbarte Proben durchschnittlich eine größere Ähnlichkeit haben als weiter entfernte.

Ein Satz von Zufallsvariablen $Z\left(x_{i}\right)$, die an jedem Punkt einer Fläche oder cines Raumes definiert sind, kann als Zufallsfunktion $Z(x)$ angesehen werden. Die Zufallsvariablen $Z\left(x_{i}\right)$ sind korreliert, die Korrelation ist abhängig von Abstand und Richtung zwischen zwei beliebigen Punkten. Eine Beobachtung $z\left(x_{i}\right)$ an einem Ort $x_{i} k a n n$ somit als eine Realisierung der Zufallsvariablen $Z\left(x_{i}\right)$ angesehen werden und ein Satz von Beobachtungen $z\left(x_{1}\right)$, $z\left(x_{2}\right), z\left(x_{3}\right), \ldots$ als eine Realisierung einer Zufallstunktion $Z(x)$. Diese Betrachtungsweise erlaubt es von ortsabhängigen (regionalisierten) Variablen zu sprechen, die durch die beiden Aspekte Zufälligkeit und Ortsabhängigkeit gekennzeichnet sind.

Die Formalisierung dieses Zusammenhangs sci zunächst am einfachen Beispiel zweier Orte mit bekanntem Abstand gezeigt. Die Zufallsvariablen $Z\left(x_{1}\right)$ und $Z\left(x_{2}\right)$ haben die Ausprägung $z_{1}$ am ersten und $z_{2}$ am zweiten Ort. Die Relation zwischen beiden Werten kann neben der Differenz $\left(z_{1}-z_{2}\right)$ durch die Varianz beschrieben werden.

$$
\begin{gathered}
s^{2}=\left(z_{1}-\bar{z}\right)^{2}+\left(z_{2}-\bar{z}\right)^{2} \\
\approx \frac{1}{2}\left(z_{1}-z_{2}\right)^{2}
\end{gathered}
$$

mit

$$
\bar{z}=\frac{z_{1}+z_{2}}{2}
$$

Beziehung (1) kann für alle möglichen Pare von Orten erweitert werden, indem die beiden Orte durch $\mathrm{x}$ und $\mathrm{x}+\mathrm{h}$ bezeichnet werden. Hierbei gibt $\mathrm{x}$ die Koordinaten eines Ortes an, $h$ ist ein Vektor, der Abstand und Richtung angibt, und als Schrittweite (englisch: $\operatorname{lng}$ ) bezeichner wird.

Für Gleichung (1) folst daher

$$
s^{2}=\frac{1}{2}\{z(x)-z(x+h)\}^{2}
$$

Wird die Betrachtung auf $m>2$ Orte ausgedehnt, die alle durch den Vektor h getrennt wercten, folgt aus (2)

$$
\vec{s}^{2}=\frac{1}{2 m} \sum_{i=1}^{m}\left\{z\left(x_{i}\right)-z\left(x_{i}+h\right)\right\}^{2}
$$

Bei der praktischen Anwentung der geostatistischen Methoden liegt ein der Stichprobenerhebung analoges Problem vor. Bei Stichproben liegt eine Realisierung $\mathrm{x}$ der Zufallsvariablen $X$ vor, wobei $x$ Teilmenge von $X$ ist. Bei Betrachtung einer Zufallsfunktion $Z(x)$ ist wiederum nur eine Realisierung $z(x)$ bchannt. Um von $z(x)$ statistische Schlußfolgerungen ziehen zu können, müssen hinsichtlich der Wahrscheinlichkeitsstruktur Annahmen getroffen werden.

Die erste Annahme besagt, daß der Erwartungswert der Zufallsfunktion unabhängig vom Ort $\mathrm{x}$ und konstant ist.

$$
E[Z(x)]=\mu
$$

Die zweite Annahme bezieht sich auf die erwartete quadratische Differenz zwischen den Werten am Ort, die durch die Schrittweite h getrennt sind.

$$
E\left[\{Z(x)-Z(x+h)\}^{2}\right]=2 \gamma(h)
$$


Dies bedeutet, daß die Varianz der Differenzen nur von h, nicht aber von $x$ abhängt, d. h. stationär für jede Schrittweite ist. Die Semivarianz $\gamma(h)$ ist der Erwartungswert der Varianz $s^{2}$ bei der Schrittweite h.

Ist das Verteilungsgesetz einer Zufallstunktion gegenüber Translationen invariant, weist die Zufallsfunktion strenge Stationarität auf. Dies bedeutet, daß die k-dimensionalen Vektoren von zwei Zufallsfunktionen $\left\{Z\left(x_{1}\right), \ldots, Z\left(x_{k}\right)\right\}$ und $\left\{Z\left(x_{1}+h\right), \ldots, Z\left(x_{k}+h\right)\right\}$ identisch sind und unabhängig von der Ausprägung des Vektors $h$. $D_{a}$ in der linearen Geostatistik nur die beiden ersten Momente betrachtet werden, genügt es, die Definition der Stationarität auf die beiden Momente zu beschräniken. Die sogenannte schwache Stationarität oder Stationarität 2. Ordnung existiert dann, wenn:

- der Erwartungswert $E\{Z(x)\}$ existiert und unabhängig vom Ort $x$ ist

- und für jedes Paar von Zufallsvariablen $\{Z(x), Z(x+h)\}$ die Kovarianz existiert und vom Vektor $h$ abhängig ist.

Die Existenz ciner Kovarianz, die zur Formulierung der Hypothese der Stationarität 2. Ordnung verlangt wird, erfordert somit eine endliche $\operatorname{Varianz} \operatorname{Var}\{Z(x)\}=C(0)$. Da Situationen, in denen keine endliche Varianz beobachtet werden kann, denkbar sind, kann die Hypothese der Stationarität 2. Ordnung zur intrinsischen Hypothese abgeschwächt werden. Die beiden in Gleichung (4) und (5) gezeigten Annahmen bilden nach Journel und Huijbregts (1978) die intrinsische Hypothese, obgleich Matheron (1965) die intrinsische Hypothese alıgemeiner formulierte. Die Konsequenzen, die aus der Nichterfüllung dieser Hypothesen resultieren, werden eingehend bei Akin und Siemes (1988, S. 189 if.) diskutiert.

Bei Gültigkeit der intrinsischen Hypothese können die Meßwerte mehrerer Paare von Proben mit Abstandsvektoren h benützt werden, um über (3) $\hat{\gamma}(h)$ zu schätzen. Die Semivarianz $\hat{\gamma}(h)$ kann für mehrere Abstandsvektoren berechnet werden, so daß eine Folge von Semivarianzen $\hat{\gamma}(1), \hat{\gamma}(2), \hat{\gamma}(3) \ldots$ resuliert. Die verallgemeinerte Gieichung zur Berechnung von $\hat{\gamma}(\mathrm{h})$ lautet

$$
\hat{\gamma}(h)=\frac{1}{2(n-h)} \sum_{i=1}^{n-h}\{z(i)-z(i+h)\}^{2}
$$

Diese sehr allgemeine Darstellung gilt nur für eindimensionale Probleme, so z. B. Bodenproben entlang einer Linie. Meist wird aber nach Zusammenhängen in einer Ebene gesucht, die eine Enveiterung der gezeigten Theorie bedingen. Wir wollen uns hier mit der gezeigten Darstellung begnïgen und auf die Literatur verweisen, da auch am eindimensionalen Fall das wichtigste Werkzeug zur Beschreibung einer Regionalisierten Variablen das Variogramm - veranschaulicht werden kann.

Werden die Schätzwerte $\hat{\gamma}(h)$ und die Abstandsvektoren $h$ in einem Diagramm aufgezeichnet, erbält man das Semivariogramm oder auch kurz Variogramm. In Abbildung 1 wird der mögliche Verlauf eines solchen Variogramms gezeigt, das zur Veranschaulichung der allgemeinen Charakteristika dienen soll. Die Varianz steigt mit zunehmender Schrittweite an bis sie ein Maximum erreicht, an dem die Kurve abflacht. Dieses Maximum wird Schwellenwert (englisch: sill) genannt. Die Schrittweite, bei der der Schwellenwert erreicht ist, wird als Reichweite (englisch: range) bezeichnet und zeigt die Grenze der räumlichen Abhängigkeit.

Eine andere Charakteristik spiegelt der Nugget-Effekt wider. Obwohl bei Schritrwei- ten, die gegen Null gehen, die Varianz ebenfalls gegen Null gehen müßte, wird oft eine deutlich größere Restvarianz beobachtet. Dicse Restvarianz - oder Nuggetvarianz - kann auf verschiedenen Ursachen wie zum Beispiel aut Meßfehlern beruhen. Häufig liegen Strukturen mit Reichweiten vor, die im Vergleich zur beobachteten Schrittweite klein sind, und nur durch zusätzliche Proben mit geringeren Abständen aufgelöst werden können. Dieses Faktum ist bei der Interpretation von Variogrammen zu beachten.

Selbstverständlich existieren auch Variogramme mit anderen, komplexeren Formen als 


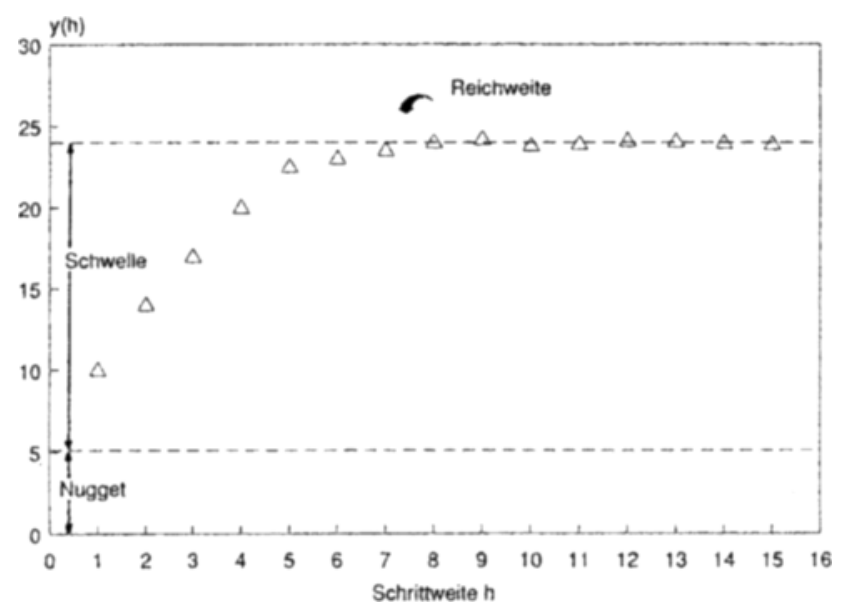

Abb. 1. Variogranm

Fig. 1. Variogram

die in Abbildung 1 dargestellten. Viele Variogramme, so auch die in dieser Untersuchung berechneten, folgen aber der hier abgebildeten Form.

Wendet man sich ausgehend vom bisher yezeigten eindimensionalen Fall der Berechnung von Variogrammen in der Fbene zu, können Variogramme für verschiedene Richtungen hergeleitet werden. Idealerweise werden Variogramme für vier Richtungen hergeleitet: für die beiden Richtungen parallel zur Ordinate und Abszisse und die beiden Diagonalen. Gleichen sich die Variogramme der verschiedenen Richtungen, so kann davon ausgegangen werden, daß die Variation richtungsunabhängig ist. Dieser Zustand wird als Isotropie bezcichnet. Weisen dic Variogramme der yerschiedenen Richtungen zwar den gleichen Schwellenwert aber unterschiedliche Reichweiten auf, spricht man von geometrischer Anisotropie. Zonale Anisotropie liegt dann vor, wenn sowohl der Schwellenwert als auch die Reichweite der Variogramme der verschiedenen Richtungen ungleich sind.

Ein weiterer Schritt bei der Anwendung geostatistischer Methoden ist die Suche nach Modellen, die die berechneten Punkte im Variogramm ausgleichen. Die Bestimmung der Ausgleichsmodelle und ihrer Parameter ist notwendig zur Herleitung lokaler Schätzwerte. Der Ausgleich kann durch die Methode der kleinsten Quadrate erfolgen. Für den Ausgleich stelen andererseits eine Reihe von Funktionen zur Verfügung, von denen hier nur das sphärische Modell, das Gausssche Modell und das lineare Modell erwähnt werden sollen. Diese Funktionen werden analog einem Handausgleich iterativ an die Punkteschar im Variogramm angepałst. Die Diskussion um die ideale Anpassung der Ausgleichfunktion ist derzeit noch kontrovers und wird sowohl von einem deterministischen wie auch empirischen Standpunkt aus geführt. Webster und Oliver (1990, S. 239 ff.) stellen die verschiedenen Ansütze und Argumentationsweisen gegenüber. In dem hier verwendeten geostatistischen Programmpaket (GEO-EAS) wird der Ausgleich interaktiv am Bildschirm vorgenommen.

Die Herleitung von Schätzwerten für Punkte oder für kleinere Flächeneinheiten (Blöcke) in einer Ebene wird durch Krigeverfahren ermöglicht, die als Schärzvorgang betrachtet werden können, bei dem eine bestimmte Anzahl von Proben innerhalb einer Nachbarschaft durch die Methode der gewichteten Mittelwertbildung einbezogen werden. Der Schätzwert für einen Block B wird durch

$$
\hat{z}(B)=\sum_{i=1}^{n} \lambda_{i} z\left(x_{i}\right)
$$


hergeleitet. Da $\Sigma \lambda i=1$ gilt, ist die Schätzung verzerrungsfrei. Die Schätzung der Varianzen von $\hat{z}(B)$, die Krige-Varianz, erfolgt durch

$$
\hat{\sigma}^{2}(B)=\sum_{i=1}^{n} \lambda_{i} \bar{\gamma}_{(}\left(x_{i}, B\right)+\psi-\bar{\gamma}(B, B)
$$

Hierbei ist $\bar{\nabla}(\mathrm{B}, \mathrm{B})$ die Varianz innerhalb des Blockes, $\psi$ ist der Lagrange - Multiplibator und $\bar{\gamma}\left(\mathrm{x}_{i}, \mathrm{~b}\right)$ ist die durchschnitcliche Semivarianz zwischen dem Block und dem i-ten Stichprobenpunkt. Die so erhaltenen Schätzwerte sind unverzerrt und haben eine minimale Varianz. Die in Gleichung ( 7 ) enthaltenen Semivarianzen werden aus der Ausgleichfunktion des Variogrammes gewonnen, womit die Bedeutung des Variogramms für die Krige-Vertahren deutlich wird.

Wir möchten die Diskussion der geostatistischen Methoden an dieser Stelle abbrechen. Neben dem hier vorgestellten linearen Krige-Verfahren für Blöcke existieren noch eine Reihe weiterer Vertahren, so z. B. das Punktkrigen oder die nichtlinearen Krige-Verfahren. Diese Verfahren und eine Vielzahl von hier nicht beschricbenen Aspekten der geostatistischen Methoden, insbesondere auch die Diskussion der Vertrauensbereiche, sind in der Literatur eingehend beschrieben.

\section{Verwendung geostatistischer Methoden in der Forstwissenschaft}

Obwohl die Theorie der Regionalisierten Variablen breiten Einfluß in der Mineralogie, der Lagerstättenerkundung und der Bodenkunde gefunden hat, finden sich vergleichsweise wenige forstiche Anwendungen.

Wraster und Oliver (1985) verwendeten geostatistische Methoden für die Bodenkartierung im Wyre Forest, England. Sie zeigten, daß die größre Variation zwischen Punkten besteht, die 66 Meter vorneinander entfernt liegen. Die solcherart korrelierten Variablen korrespondierten mit dem Vorkommen bestimmter Bodentypen.

Ein Ansatz basierend auf geosratistischen Methoden zur Kartierung von Plantagenflächen für die Behandlung mit unterschiedlichen Düngergaben wurde von PAYN und Clougr (19S8) vorgestellt. Die räumliche Variabilität von Schwermetallen in einem ein Hektar großen Bestand wurde durch die Berechnung von Variogrammen von Wopereis et al. (1985) beschrieben.

Palmer (1988) beschreibe mit einer Kombination von Ordination und geostatistischer Methoden die räumliche Struktur von Pflanzengesellschaften und leitet darauf aufbauend verschiedene Quadratgrößen und Probenabstände für vegetationskundliche Untersuchungen her.

Ramirez-Maldonado (1988) hat die Theorie und die Methode der Geostatistik im Kontexi von Forstinventuren diskutiert. Bei der Annahme, die Beobachtungen auf den Stichprobenflïchen seien unabhängig, wird die räumliche Beziehung zwischen den Proben ignoriert, obwohl bei nahe beieinander liegenden Probetlïchen sehr of Korrelationen beobachtet werden können. Das Variogramm der Grundflächenmessung mit Hilfe der Winkelzählprobe wurde von Ramirez-Maldonado durch ein sphärisches Modell ausgeglichen und daraus die Fmpfehlung abgeleitet, daß kleinere Zählfaktoren effizienter sind als große. Er hat geostatistische Methoden ebenfalls zur Analyse der 10-Punkt-Cluster des US-Forest-Service und für zweistufige Stichprobenverfahren angewendet. Eine neue theoretische Betrachtung für die klassische Winkelzälalprobe wird präsenticr, welche die Punktstichprube als multinomialen Prozeß betrachtet.

Die Möglichkeiten der räumlichen Interpolation mir Hilfe von Krige-Verfahren zur kartographischen Darsteliung von Forstinventurdaten wurde von SAMRA et al. (1989) am Beispiel des Höhenzuwachses von Molia Azederach in Karnal, Indien untersucht. Die Baumhöhe varierte beim untersuchten Datenmaterial sowohl in Abhängigkeit von der 
Schrittweite $h$ als auch von der Richtung innerhalb des Bestandes (Anisotrophie). In Abhängigkeit vom Alter konnten über $70 \%$ der Heterogenität der Baumhöhe in der Nordwest-Südostrichtung erklärt werden.

\section{Anwendungsbeispiel Waldschadeninventur 1986-1990}

Um die Eignung geostatistischer Methoden zur Analyse von Inventurdaten und die sich damir eröffnenden Möglichkeiten für ein umfassendes, ursachenklärendes Monitoring abzuschätzen, wurden die Daten der Waldschadeninventur 1986 und 1990 herangezogen. Die Wahl des ältesten und des jüngsten Inventurzeitpunktes, für die konsistente Daten vorlagen, soll zudem die Praktikabilität zur Beschreibung von Zustandsveränderungen aufzeigen.

Bei der Auswertung wurden alle Büume berücksichtigt, für die eine Nadel-/Blattverlustschätzung vorliegt. Fine Untergliederung in einzelne Baumarten erfolgte nicht. Wie in Abschnitt 2 dargestellt wurde, betrachten die geostatistischen Merhoden Beobachrungen an Punkten. Da die Daten der Waldschadeninventuren aber aut Flächen erhoben werden, muß eine Zusammenfassung der Daten über die Mittelwertbildung erfolgen. In der Geostatistik wird der Mittelwert $z_{v}(x)$ als die Regularisierung der Punktvariablen $z(x)$ über dem Träger v(x) (engl.: support) bezeichnet, d. h. im hier gezeigten Fall über die Fläche des Probekreises.

Die in 5-\%-Stufen ertolgten Nadel-/Blattverlust-Schätzungen (NBV) der Einzelbäume wurden probeflächenweise zusammengefaßst und analog den jährlichen Auswertungen der Waidschadeninventur für die "Sanasitva" Waldschadenberichte nach

$$
\overline{\mathrm{NBV}}=\frac{\sum \mathrm{NBV} \mathrm{Bhd}_{\mathrm{i}}{ }^{2}}{\Sigma \mathrm{Bhd}_{\mathrm{i}}{ }^{2}}
$$

mit dem Bhd ${ }^{2}$ gewichtet. In Abbildung 2 sind die Rohdaten für die Jahre 1986 und 1990 dargestellt. Es fällt eine Zunahme der Nadel-/Blartverluste zwischen 1986 und 1990 auf, wenn auch aus der hier gezeigten Darstellung noch keine gesicherten Rückschlässe aut die räumliche Verteilung der Waldschäden gezogen werdèn können.

Die geostatistischen Auswertungen wurden mit dem Programmpaket GEO-EAS (Geostatistical Environmental Assessment Software) durchgeführt, das freundlicherweise von E. Englund und A. Sparks von der US Environmental Protection Agency, Las Vegas, zur Verfügung gestellt wurde.

Wie in Abschnit 2 beschrieben, wurden für jeden Rohdatensatz zunächst Variogramme hergeleitet. Aufgrund der Verteilung der WSI-Probeflächen in einem $4 \times 4 \mathrm{~km}$ Netz waren dic möglichen Entfernungen auf $4 \mathrm{~km}, 5,65 \mathrm{~km}, 8 \mathrm{~km}, 11,3 \mathrm{~km}, \ldots$ begrenz. . Da 1990 zusätzlich Proben zufälig in das $4 x+\mathrm{km}$ Netz gelert wurden, waren hier Entfernungen von $1 \mathrm{~km}, 1,4 \mathrm{~km}, 2 \mathrm{~km}, 2.8 \mathrm{~km}, \ldots$ realisierbar. Diese relativ großen Abstände zwischen den Probetlächen führen zwangsläufig zu hohen Nuggetcffekten. Kleinräumige Aggregationen von Nadel-/Blattverlusten können hierbei nicht mehr aufgedeckt werden, obwohl diese bei der Betrachtung lokaler Expositionen oder der Auswirkung lokaler Standortsunterschiede ein wichriger Indikator für Ursachen-/Wirkungskomplexe sein könnten.

Dieser Zusammenhang stellt ein generelles Problem bei der Anwendung geostatistischer Methoden bei Großrauminventuren dar. Während bei Bestandesinventuren durchaus Abhängigkeiten zwischen benachbarten Proben vorstellbar sind, ist dies bei großräumigen Erhebungen mit notwendigerweise großen Abständen zwischen den Proben nur für eine beschränkte Anzahl von Variablen denkbar. So zeigten sich beispielsweise bei der Untersuchung der Volumenwerte der I.FI-Probeflächen des Berner Obcrlandes keine räumlichen Abhängigkeiten. Dies ist leicht nachvollziehbar, da es keine rationale Begründung dafür gibr, daß in bewirtschatteten Wäldern mit kleinflächigen Beständen zwei Probetlächen, die 


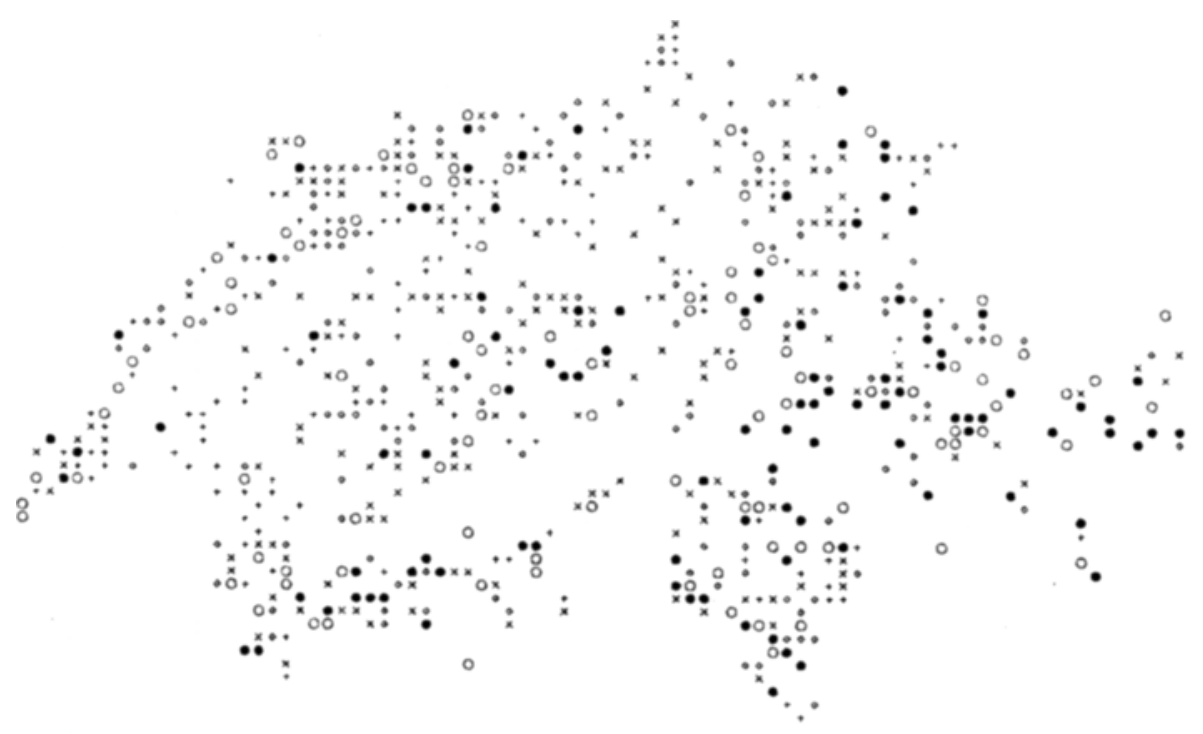

Verlust $+++<10 \% \times \times \times 10-14 \% \diamond \diamond \diamond 15-19 \%$

$\circ \bigcirc \bigcirc 20-24 \% \bullet \bullet \bullet>=25 \%$

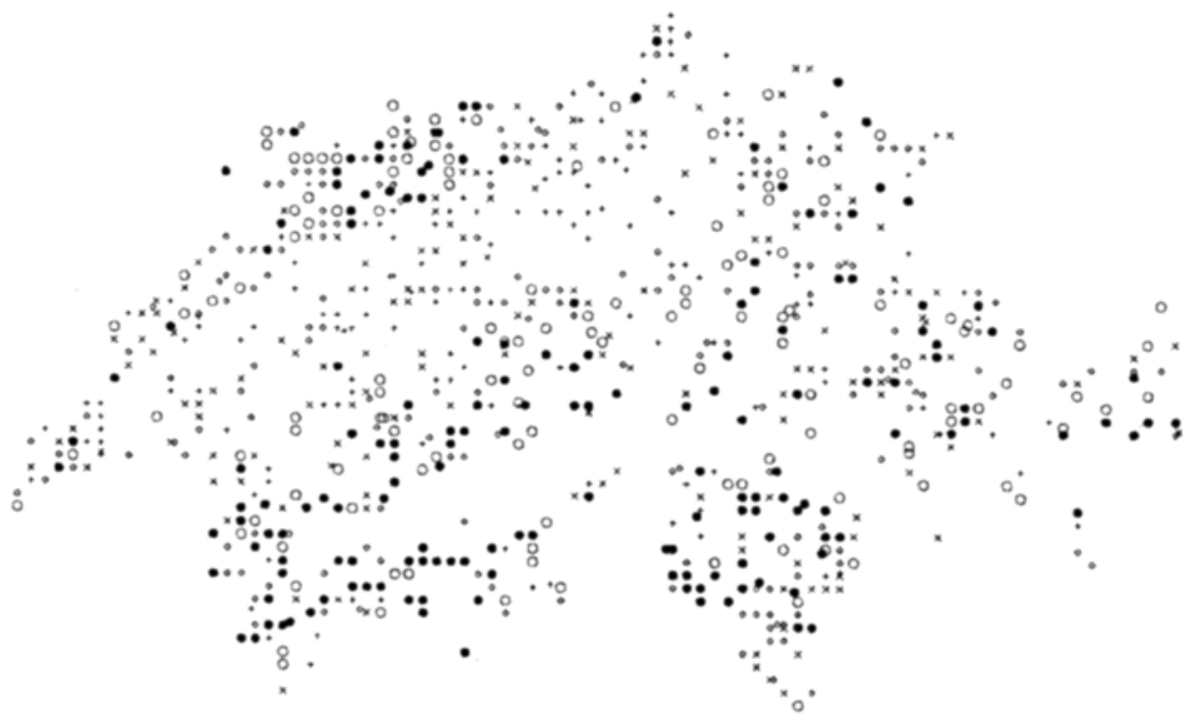

Verlust $+++<10 \% \times \times \times 10 \cdot 14 \% \diamond \diamond \diamond 15-19 \%$

○० $20-24 \% \bullet \bullet \bullet>=25 \%$

Abb. 2. Rohdaten. Oben: 1986, unten:1990

Fig. 2. Raw da:a. Above: 1986, below 1990

einen Kilometer weit auseinander liegen, ein „ähnlicheres" Volumen haben sollen als weiter entfernte Probetlächen.

Eine Anwendung der geostatistischen Methoden auf WSI-Daten erscheint weniger problematisch, da regionale Unterschiede der Nadel-/Blattverluste bekannt sind und somit 
auch bei größeren Abständen zwischen den Probeflächen gefunden werden können. Die Anpassung von Modellen an die Variogramme war nicht ohne Probleme möglich. Für die Jahre 1987, 1988 und 1989 konnten keine Modelle zur Beschreibung räumlicher Abhängigkeiten angepaßt werden. Da auch für die Jahre 1986 und 1990 Modelle nur mit großzzügigen Zugeständnissen angepaßt werden konnten, wurde auf die Herleitung richtungsabhängiger Variogramme verzichter. Die folgenden Diskussionen und Interpretationsmöglichkeiten erfolgen unter der Annahme der Gültigkeit der Varianzen und Modelle und dienen vorwiegend zum Aufzeigen der Erklärungsmöglichkeiten bei der Anwendung geostatistischer Methoden zur Beschreibung räumlicher Verteilungen. Bei der Analyse der Variogramme kann sowohl für 1986 als auch für 1990 eine Reichweite von $8 \mathrm{~km}$ angenommen werden. Aufgrund dieser geringen Reichweiten lagen nur wenige Punkte zur Anpassung der Modelle und zum Auffinden der Nuggetvarianzen vor.

Tabelle' 1. Ausgleichsfunktionen

Table 1. Adjustment functions

\begin{tabular}{|cccccc|}
\hline Bammart & Jahr & Wodell & Nugget & Schwelle & Reichweite \\
\hline Alle & 1986 & Exponential & 50,0 & 30,0 & 8,0 \\
Aile & 1990 & Exponential & 30,0 & 65,0 & 8,0 \\
\hline
\end{tabular}

1986 lag der Nuggeteffekt bei 50,0 und der Schwellenwert bei 30. Während die Reichweite gleich blieb, erfolgte 1990 eine Umkehrung der Größenordnung des Nuggeteffektes $(=30)$ und des Schwellenwertes $(=65)$. Als Ursache für diese Umkehrung sind verschiedene Faktoren vorstellbar.

Während 1986 die Aufnahmeequipen in 5 großen, zusammenhängenden Gebieten eingesetzt wurden, erfolgte 1990 cine häufige Umsetzung der Gruppen. Der gegenüber 1986 geringere Nuggeteffekt kann auf eine Aggregation der Gruppeneffekte hindeuten. Verschicdene Einflüsse, wie z. B. die Wettersituation während mehreren, zusammenhïngenden Aufnahmetagen oder das unterschiedliche Niveau bei der Nadel-iBlattverlustschätzung in Abhängigkeit von den vorher besuchten Gebieten können zu einer scheinbaren, Jokalen Abhüngigkeit der Nadel-/Blattverluste führen.

Eine andere Frklärung für den geringeren Nuggeteffekt könnte in einer verbesserten Schulung der Aufnahmegruppen liegen. Die Gruppen werden mittlerweile bei jedem neuen Aufnahmeturnus, d.h. der Lmsetzung in eine neue Aufnahmeregion, speziell für die Nadel-/Blattverlustschätzung in diesen Gebieten geschult. Die Schulungsmaßnahmer können dazı geführt baben, daß gegenüber 1986 eine geringere „ungeklärte“ Varianz auftrat. Da sich dieser unerklärte Varianzanteil in Nuggeteffekt widerspiegelt, könnte auch dieser Zusammenhang eine Erklärung für die Verringerung des Nuggeteffektes sein.

Diese beiden Erklärungsyersuche tühren zu einer Siruation, in der die lokalen Abhängigkeiten der Nadel-/Blattverluste durch das Schätzverhalten der Gruppen lediglich vorgetäuscht werden. Andererseirs können sich aber die räumlichen Abhängigkeiten mit der Zeitentwicklung ausgeprägt haben. Dieser Erklärungsansatz erscheint sowohl bei der Betrachtung lokaler Schadstoffemittenten als auch klimatischer Faktoren als Ursache für den Gesundheitszustand der Walder plausibel. In Abhängigkeit von Standortsfaktoren oder Variationen von Baumarten und Phänotypen ist eine zeitlich verzögerta Reaktivität denkbar, die bei einer genügend langen Exposition aber den gleichen Effeht - nämlich ein crhöhter Nadel-/Blattverlust - zeigen kann.

Welches dieser Erklärungsmodelle zutrifft und welche Ursache zur beobachteten Aggregation führt, kann anhand der vorliegenden Daten und Modelle nicht überprüft werden. Die Zunahme der Gesamtvarianz deutet auf eine Erthöhung der Schäten auf 
einzelnen Probeflächen hin. Dies erlaubt den Rückschluß, daß die Nadel-/Blatvverluste beim Vergleich der Jahre 1986 und 1990 heute insgesamt aggregierter aber mit einer größeren Spannweite vorzukommen scheinen.

Lokale Schätzwerte wurden durch zweidimensionales Block-Krigen hergeleiter. Die Ergebnisse sind in Abbildung 3 dargestellt. Erkennbar ist die Zunahme der Regionen, in denen höhere Nadel-/Blattverluste geschätzt wurden. Im Engadin ist eine Abnahme der Nadel-/Blattverlust-Schätzung zu beobachten. Der Vergleich mit den in Abbildung 3 dargestellten Rohdaten zeigt, wie die Einbeziehung benachbarter Proben die lokalen Schätzungen beeinflussen.

\section{Diskussion der Ergebnisse}

Die dargestellten räumlichen Verteilungen können nur bei Beachtung zusätzlicher Gegebenheiten interpretiert werden. So ist bei jedem statistischen Schätzverfahren neben dem Schätzwert der Schätzfehler zu berücksichtigen. Besonders in Randbereichen und in Gebieten, die durch wenige Probeflächen abgedeckt sind, erreichen die Standardfehler Werte, aufgrund welcher die Interpretation der räumlichen Verteilung der Schäden und insbesondere der Zustandsveränderungen mit Vorsich erfolgen muß.

Neben den Standardfehlern der Schätzung werden die Resultate von weiteren Fehlerquellen beeinflußt (siehe hierzu auch Gertver u. KöHL 1992). Die Schätzung der Nadel-/ Blattverluste wird europaweit praktiziert und ist das derzeit von der ECE favorisierte Verfahren zur Beurteilung der Vitalität des Einzelbaumes, obwohl die Problematik der Fehler, die bei der Beurteilung der Nadel-/Blattverluste auftreten, bekannt ist. KubliN (1987) konnte für die baden-württembergische Waldzustandsinventur die Effekte von Aufnahmeequipen nachweisen. Kö Hr (1991) hat anhand der Daten der schweizerischen WSI durch ein logistisches Modell gezeigt, daß neben den Gruppeneinflüssen zusätzliche Effekte wie das Wetter, die Entwicklungsstute der Bestände und die soziale Stellung des Einzelbaumes die Nadel-/Blattverlust-Schätzung beeinflussen.

Die geostatistischen Methoden gehen - wie alle interenzstatistischen Verfahren, die zur Auswertung der WSI-Daten angewendet werden - von der Annahme fehlerfreier Beobachtungen aus. Zwar wird bei der WSI versucht, durch verschiedene Malinahmen wie die Vorgabe einer konstanten Ausprechrichtung der Bäume oder die obligatorische Benutzung des Kronenbilderbuches (Müller u. Stierlin 1990) eine Vermeidung subjekriver Beobachrungen und die Reproduzierbarkeit der Schätzungen zu erreichen, dennoch können die Nadel-/Bhattverlust-Daten nicht als fehlerfreie Meßgrößen angesehen werden. Der Einfluß dieser Fehter sowie die Auswirkungen von Ausreißern auf die lokalen Schätzungen mir Hilfe geostatistischer Methoden bleibt noch zu überprüfen.

$\mathrm{Da}$ durch die organisatorische Notwendigkeit, die Inventurgruppen in größeren, zusammenhängenden Gebieten einzusetzen, die Beobachtungsfehler ihrerseits eine räumliche Tendenz autweisen können, sind die in Abbildung 3 dargestellten räumlichen Verteilungen nur bei Beachtung der Einsatzgebiete der Aufnahmegruppen zu interpretieren. Räumliche Trends, die 1990 beobachtet wurden, sind nicht mehr auf einzelne Gruppen zurückzuführen, sondern zeigen sich bei mehreren Gruppen. Die starken Nadel-/Blattverluste im Nordwesten der Schweiz überdecken beispielsweise die Einsatzgebiete von vier Feldaufnahmeequipen.

Die Qualität der mit den Krigeverfahren hergestellten Karten hängt ursächlich mit der Anpassungsgüte der Modelle an die Variogramme ab. Die geringen Reichweiten, die in dieser Untersuchung gefunden wurden, haben zur Folge, daß nur eine limitierte Anzahl von Abständen mit zum Teil wenigen Beobachtungen für die Anpassung der Modelle zur Vertügung standen. Daraus resultieren Unsicherheiten bei der Modellwahl und der Festlegung der Nuggetvarianz. 


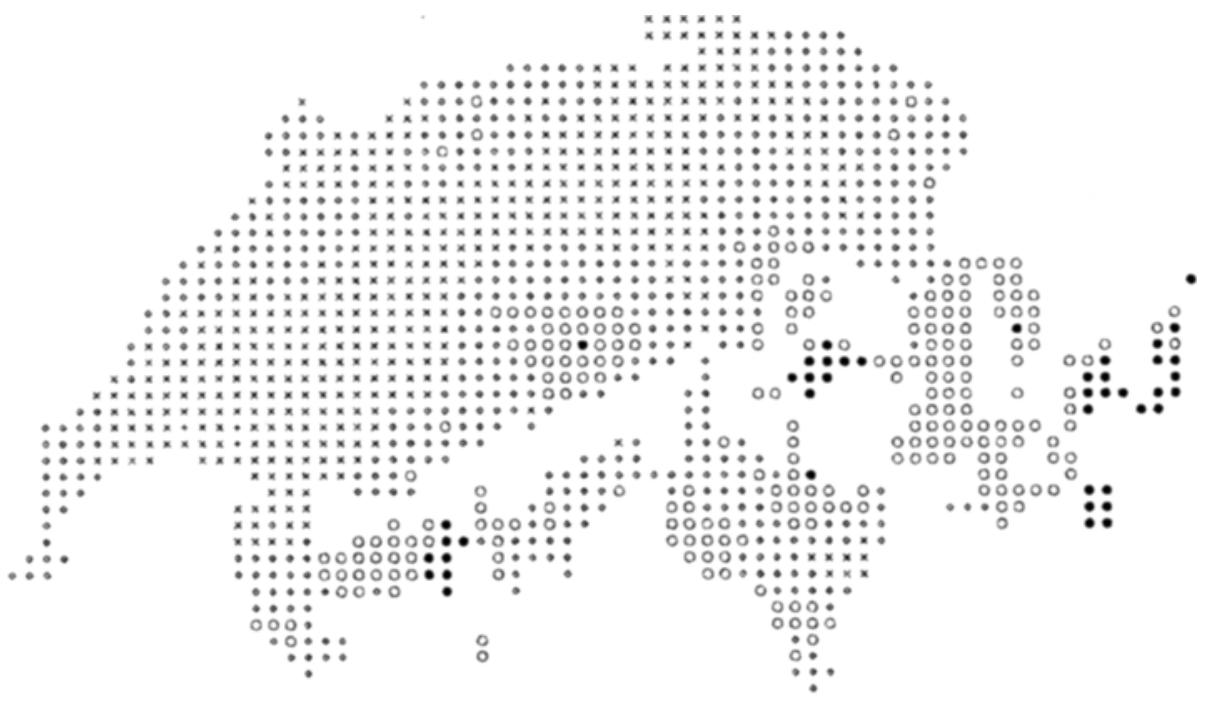

Verlust $+++<10 \% \times \times \times 10-14 \% \diamond \diamond \diamond 15-19 \%$

$0 \bigcirc 020.24 \% \bullet \bullet \bullet>=25 \%$

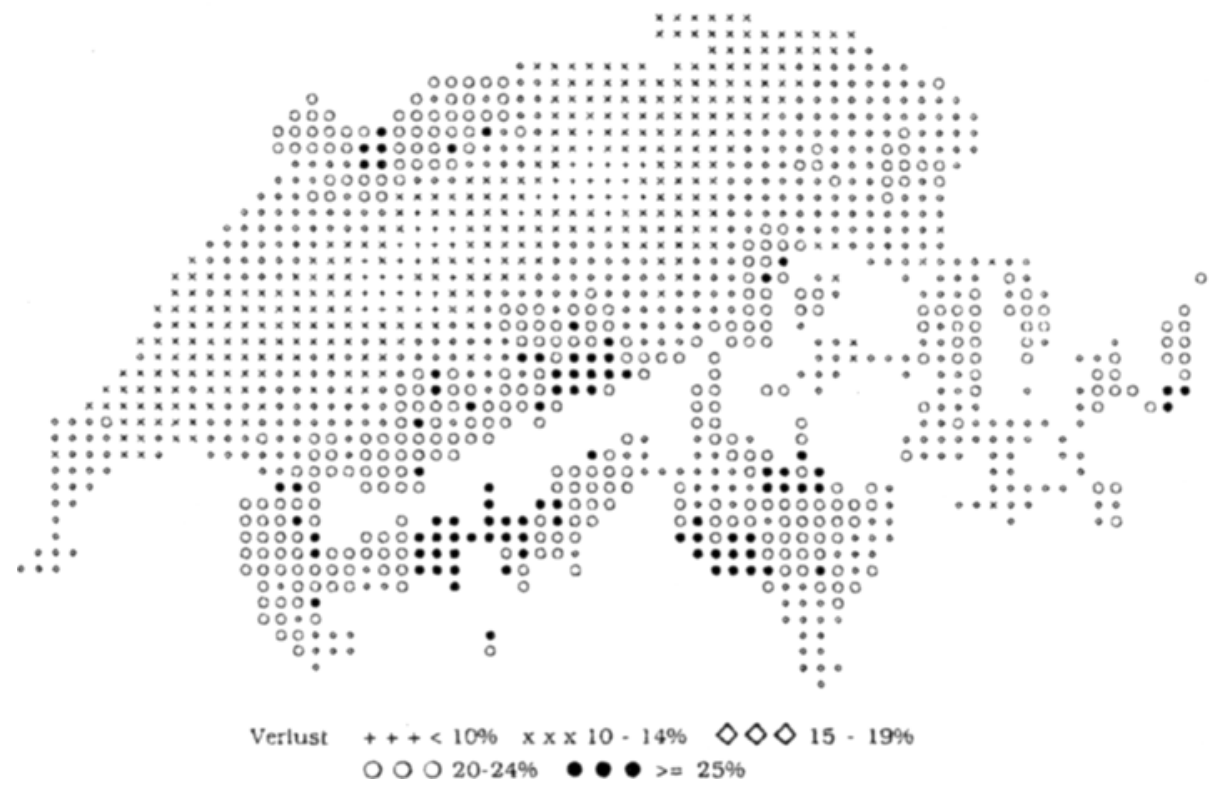

Abb. 3. Käumliche Verteilung der Nadel-/Blattverluste (Kriging-Schätzwerte) Oben: 1986, unten: 1990

Fig. 3. Spatial distribution of crown desity (Kriging estimates). Above: 1986, below: 1990

Die hier gezeigte Diskussion ist nur unter der Annahme der Gültigkeit der gefundenen Varianzen und Modelle möglich und erhebt somit nicht den Anspruch auf Allgemeingültigkeit. Sie soll vielmehr dazu dienen, die prinzipiellen Möglichkeiten und das Erklärungspotenial geostatistischer Methoden aufzuzeigen. 


\section{Ausblick}

Die hier gezeigte Methode ist trotz der angeführten Vorbehalte im Ansatz geeignet, die räumliche Verteilung der Waldschäden zu beschreiben. Insbesondere beim Vergleich verschiedener Inventurzeitpunkte und der getrennten Behandlung verschiedener Baumarten bilden geostatistische Methoden ein geeignetes Instrument epidemiologischer Untersuchungen.

Die Probleme bei der Modellanpassung erfordert allerdings weitere Untersuchungen, wobei insbesondere die Sensitivität der Krigeverfahren bezüglich fehlerhafter Modelle zu überprüfen ist. Auch die Beziehung zwischen Probeflächenabstand, räumlicher Variation und Größe der Aussagecinheit sowie dic Betrachiung der Anisotropie und das Schätzverhalten bei der Anwendung der fortgeschrittenen Methoden der Geostatistik (UniversalKrigen) müssen im Kontext großräumiger Erhebungen diskutiert werden.

Impulse für die Ursachenforschung im Bereich der Waldschäden können aus dem Zusammenspielen der räumlichen Verteilung der Waldschäden und anderen Daten mit räumlichem Bezug folgen. So können mit der hier vorgestellten Methode analog Karten für die Schadstoffbelastung der Luft und des Bodens oder Klimadaten erstellt und mit Waldschadenkarten verolichen werden. Dieser vielversprechende Ansatz kann zur Zeit allerdings nicht weiter verfolgt werden, da in der Schweiz - wie in fast allen europäischen Ländern - entsprechende Meßsstationen nicht errichtet sind und folglich wichtige Daten zur Herlcitung kausaler. Zusammenhänge fehlen.

Die Festlegung der Standorte von Mießstationen oder Dauerbeobachrungsfiüchen kann anhand von Verteilungskarten der Nadel-/Blattverluste oprimiert werden. Ebenso kann mit Hilfe der Verteilungskarten eine Auswahl der WSI-Probeflächen erfolgen, an denen intensivere Beobachtungen, z. B. Boden- und Nadelproben erfolgen sollen.

Der Vergleich der räumlichen Verteilung der Nadel-/Blattverluste mehrerer Jahre kann zur Aufdeckung unplausibler, kurzfristiger Veränderungen dienen und damit Hinweise für die Güte der Nadel-/Blatverlust-Schätzungen in einzelnen Jahren oder bei einzelnen Gruppen geben.

Der hier vorgestelle Versuch zur Beschreibung der räumlichen Verteilung der Waldschäden sowie die Optionen, die aus der Anwendung geostatistischer Verfahren erwachsen, stimmen optimistisch und rechtertigen weitere Untersuchungen. Die gezeigten Methoden, Ergebnisse und Perspektiven machen aber vor allem deutlich, daß die Waldschadeninventuren trotz der häufig geäußerten Kritik nicht in eine Sackgasse getührt haben, sondern einer der ersten Schritte zu einem integrierten Umweltmonitoring sein können.

\section{Zusammenfassung}

Die räumliche Darstellung der Waldschäden durch die Erstellung einer einfachen Karte der beobachteten Nadel-/Blattveriuste im Srichprobennetz führt zwangsläufig zu Fehlinterpretationen, da die erhobenen Proben im Wald nicht repräsentativ für die sie umgebende Fläche sind und nicht unterschieden werden kann, ob die Lokalisation eines Nadel-/Blattverlustes einen regionalen Trend oder eine zufällige Beobachtung innerhalb der kleinräumigen Variation darsielic. Geostatistische Methoden wurden für ähnliche Probleme in Bereich der Mineralogie entwickelt und werden hier angewendet, um ausgehenù von dem durchschnittlichen Nadej-/Blattverlust einzelner Probeflächen dic räumliche Verteilung der Nadel-Blattverluste darzustellen. Die geostatistischen Methoden werden kurz vorgestellt, wobei besonders auf die Herleitung des Variogrammes eingegangen wird, und anhand der Daten der Schweizer Waldschadeninventur 1986 und 1990 die Anwendungsmöglichkeiten beschrieben werden.

Geostatistische Methoden bilden ein geeigneres Instrunent für epidemiologische Lntersucnungen. Durch das Zusammenspielen räumlicher Verteilungen der Waldschäden und anderen Daten mit räumlichem Bezug können Impulse für die Ursachenforschung gewonnen werden. Der Vergleich der räumlichen Verteilung mehrerer Jahre kinn zur Aufdeckung auffallender, kurzfristiger Verändenngen dienen. 


\section{Summary}

Application of geostatistical methods for the analysis of forest danage inventories: Possibilities for the description of sputial distributions

The spatial distribution of forest damage by using simple maps of the observed needle/leaf loss from the sampling grid can easily lead to misinterpretations, because needle/leaf losses on sample plots are not representative for the surrounding area. Otien it can not be decided if the localization of a single needle/leaf loss is due to a local trend or a random observation within the normal spatial variation. Geostatistical methods are applied in this paper to show the spatial distribution of needle/leaf losses based on the arerage needle/leaf loss of individual plots.

Geostatistical methods are briefly described, with special emphasis on the construction of variograms. Applicability is shown on a data set of the Swiss forest damage assessments in 1986 and 1990.

Geostatistical methods are an ideal tool for epidemiological studies. By combining the spatial distribution of forest damages with other spatially related statistics, new relacions in causal rescarch can be found. The comparison of spatial distribution of several years can reveal significant short-term changes.

\section{Literatur}

Akin, H.; Siemrs, H., 1988: Praktische Geostaristik, Springer Verlag, Heideiberg.

Clark, 1., 1979: Practical Geostatistics, Applied Sciences Publishers, London.

Davio, 11, 1977: Gecstatistical Ore Reserve Estimation, Elsevier, Amsterdam.

Englund, E.; Sparks, A., 1988: GEO-EAS (Geostatistical Environmental Assessment Software), User's Guide, Environmental Monitoring Systems Laboratory, U.S. EPA, Las Vegas.

GertNer. G.; Köhl, M., 1992: An assessment of some nonsampling errors in a national survey using error budgets. For. Sci. 38, 3, 525-53S.

Jolriel, A. G.; Hujeregts, Ch. J., 1978: Mining Geostatistics, Academic Press, London.

KöHL, M., 1991: Waldschadensinventuren: mögliche Ursachen der Variation zwischen Beobachtern bei der Nadel-/Blattverlustschätzung und Folgerungen für Kontrollaufnahmen. AFJZ 162, 11/12: $210-221$.

Krige, D. G., 1951: A Statistical Approach to Some Basic Mining Valuation Probiems on the Witwatersrand. J. Chem. Metall. Min. Soc. S. Africa 52, 6, 119-139.

-.. 1966: Two-dimensional weighted mosing average trend surfaces for ore-evaluation. Journal of the South African Institute of Mining and Metallurgy 66, 13-38.

Kubliv, E., 1987: Statistische Auswertungsinodelic für Waldschadensinventuren - Methodische Úberlequngen. Forstw. Cbl. 106, 57-68.

Müller, F.; Srierlin, H. R., 1990: Sanasilya Kronenbilderbuch, Eidgen. Forschungsanse. Wald, Schnee und Landschaft.

PaLAER, M. W., 1988: Fractal geometry: a cool for describing spatial patterns of plant communities. Vegetatio $75,1-2,91-102$.

PaYn, $T$. W.; Clougri, M. E., 1988: Differential fertilisation on pine plantations on acid furest soils, South Africin Forestry Journal 147, 16-25.

Ramirez-Natdonado, H., 1989: On the relevance of geostatistical theory and methods to forest inventory problems, Ph. D. Thesis, Univ. of Georgia.

Samra, J. S.; Gill, M. S.; Bhatra, V. K., 1989: Spatial stocinastic modeling of growth and forest resource evaluation. For sci. 35, 3,63j-676.

Wesster, R.; Olivier, M. A., 1985: Utilisation exploratoire de ha geostatistique pour la cartographie du sol dans la foret de Wyre (GB). Sciences de La Terre. Informatique Geologique 24, $171-173$.

- 1990: Statistical methods in soil and land resource survev. Oxford University Press, Oxtord.

Worereis, M. C.; Gascuel-Odoux, C.; Bolrrie, G.; SolGnet, G., 1938: Spatial variability of heary metals in soil on a onc-hectare scale, Soil Sciences $146(2): 113-118$.

Anjcbriften der Verfasser: Dr. MrсHaEl KöHL, Eidgenössische Forschungsanstalt für Wald, Schnee und Landschaft, Gruppe Inventurmethode, 8903-Birmensdorf, Schweiz; Dr. Grorge GerT:NER, University of Illinois, Department of Forestry. Urbana/Iltinois. USA. 